\title{
Síndrome de quebra-nozes (nutcracker) em uma mulher jovem em investigação por quadro de hematúria recorrente: um relato de caso
}

Nutcraker syndrome as a cause of recurrent hematuria in a young woman: a case report

\begin{abstract}
Autores
Gustavo Lenci Marques José Gastão Rocha de Carvalho

Marcelo Mazza do Nascimento

Sérgio Godoy Marks

Luís Guilherme Olbertz

Universidade Federal do Paraná (UFPR).
\end{abstract}

Data de submissão: 08/05/2011 Data de aprovação: 09/06/2011

Correspondência para: Gustavo Lenci Marques Serviço de Nefrologia Departamento de Clínica Médica da UFPR

Rua General Carneiro, 181

- $10^{\circ}$ Andar

Centro

Curitiba - PR - Brasil.

CEP 80060-900

E-mail:

gustavolencimarques@

yahoo.com.br

O referido estudo foi realizado no Departamento de Clínica Médica - Serviço de Nefrologia da UFPR -

Curitiba - PR - Brasil.

Os autores declaram a inexistência de conflitos de interesse.

\section{Resumo}

Introdução: A síndrome de quebra-nozes (SQN) é uma condição na qual há compressão da veia renal esquerda pela aorta e a artéria mesentérica superior, associada a uma sintomatologia característica, porém pouco específica. Relato do caso: Trata-se de uma paciente de 37 anos, apresentando hematúria macroscópica intermitente sem etiologia estabelecida até então. Durante a investigação, uma angiotomografia evidenciou compressão da veia renal esquerda no seu trajeto entre a artéria mesentérica superior e aorta abdominal, associada a importante dilatação de veia ovariana esquerda. Discussão: A síndrome desencadeada pela compressão da veia renal esquerda pela aorta e artéria mesentérica superior apresenta prevalência provavelmente aumentada no sexo feminino e postula-se que seja associada a situações particulares, como a nefroptose e a escassez de gordura retroperitoneal. Hematúria, sinal típico, teria como causa a ruptura do fino septo que separa as veias do sistema coletor urinário. Vários exames de imagem podem auxiliar no diagnóstico. O tipo de tratamento que deve ser adotado ainda é controverso, existindo diversas opções cirúrgicas, como o uso de stent intravascular e a conduta conservadora. De diagnóstico costumeiramente tardio, essa síndrome deve ser incluída na investigação de pacientes com quadro de hematúria a esclarecer.

Palavras-chave: Hematúria. Dor abdominal. Artéria mesentérica superior.

\section{Abstract}

Introduction: The nutcracker syndrome is defined by the compression of the left renal vein between the aorta and superior mesenteric arteries, accompanied by a classic, but less specific, symptomatology. Case report: We reported a case of a 37-year-old woman who had been investigated because of intermittent gross hematuria of unknown origin. Computed tomographic angiography revealed compression of the left renal vein as it ran between the superior mesenteric artery and the abdominal aorta, associated with significant dilation of the left ovarian vein. Discussion: The nutcracker syndrome, caused by compression of the left renal vein by the aorta and superior mesenteric arteries, is probably more prevalent in women, and it is caused by specific situations, such as renal ptosis and paucity of retroperitoneal fat. Hematuria, a typical symptom, is due to rupture of the thin-walled septum separating the veins of the urinary collecting system. Imaging tests may help with the diagnosis. The best treatment is still elusive, but there are several surgical approaches, such as intravascular stenting, and also a conservative management. Diagnosis is usually delayed, and the syndrome should be included in the differential of hematuria of unknown origin.

Keywords: Hematuria. Abdominal pain. Mesenteric artery, superior. 


\section{INTRODUÇÃO}

A síndrome de quebra-nozes ( $\mathrm{SQN}$ ) é uma condição de diagnóstico difícil e comumente tardio, ${ }^{1}$ na qual há compressão da veia renal esquerda pelas artérias aorta e mesentérica superior. É caracterizada tipicamente por hematúria, ${ }^{2}$ porém outros sinais e sintomas podem ocorrer, como dor pélvica, varicocele esquerda e proteinúria ortostática. O objetivo deste relato foi descrever um caso de SQN em uma paciente com quadro de hematúria macroscópica recorrente, mostrando sua evolução clínica, exames laboratoriais e revisando dados da literatura, a fim alertar ao nefrologista sobre a possibilidade diagnóstica dessa síndrome, ao se deparar com pacientes em investigação por um quadro de hematúria a esclarecer.

\section{Relato do caso}

Paciente V.W.G., 37 anos, feminino, branco, natural de Curitiba (PR). Foi encaminhada ao nefrologista por quadro de hematúria a esclarecer. Relatou que, em fevereiro de 2009, apresentou seu primeiro episódio de hematúria macroscópica, na época tratada como infecção do trato urinário. Dois novos episódios de hematúria ocorreram em setembro de 2009 e em março de 2010. Entre outras queixas, tinha insônia de longa data e, ocasionalmente, sensação desagradável de calor na região genital. Apresentava antecedente de três gestações, com um aborto e duas cesarianas. Sem história familiar de hematúria ou doença renal na família. Negava tabagismo ou etilismo.

Ao exame físico apresentava-se lúcida, com pressão arterial de 100 x $60 \mathrm{mmHg}$ (sentada) e 96 × $70 \mathrm{mmHg}$ (em pé), frequência cardíaca de $78 \mathrm{bpm}$ e peso de $62 \mathrm{~kg}$.

Exames laboratoriais, radiológicos e endoscópicos: parcial de urina (fevereiro de 2009) apresentou hematúria macroscópica, sem proteinúria, sendo sua creatinina sérica de 0,62 $\mathrm{mg} / \mathrm{dL}$. Ultrassonografia de abdome (outubro de 2009) e cistoscopia (novembro de 2009) apresentaram resultados normais. Uma angiotomografia

Figura 1. Compressão da veia renal esquerda entre a mesentérica superior e a aorta (circulado).

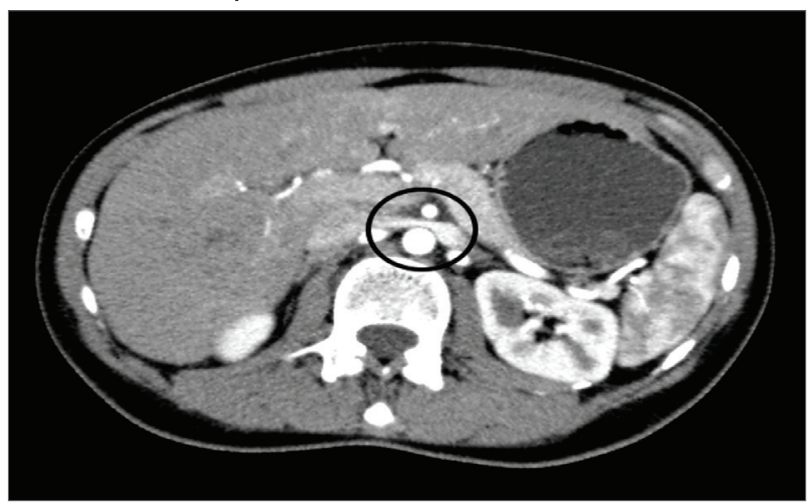

Figura 2. Compressão da veia renal esquerda entre a mesentérica superior e a aorta (na região circulada).

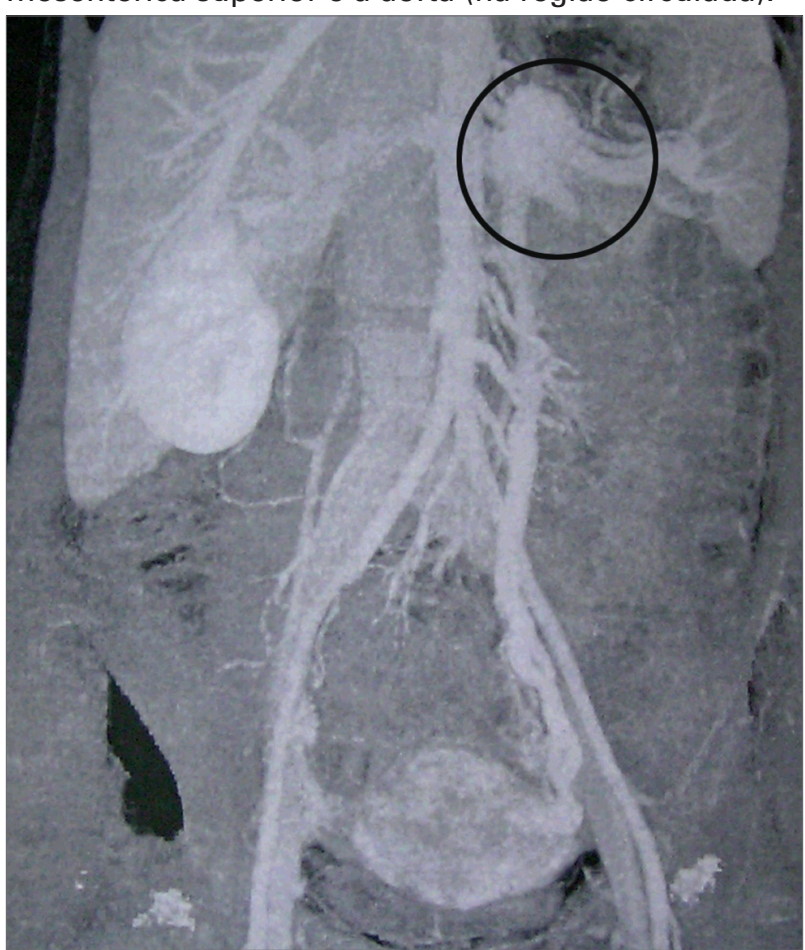

Figura 3. Congestão da veia gonadal à esquerda (seta).

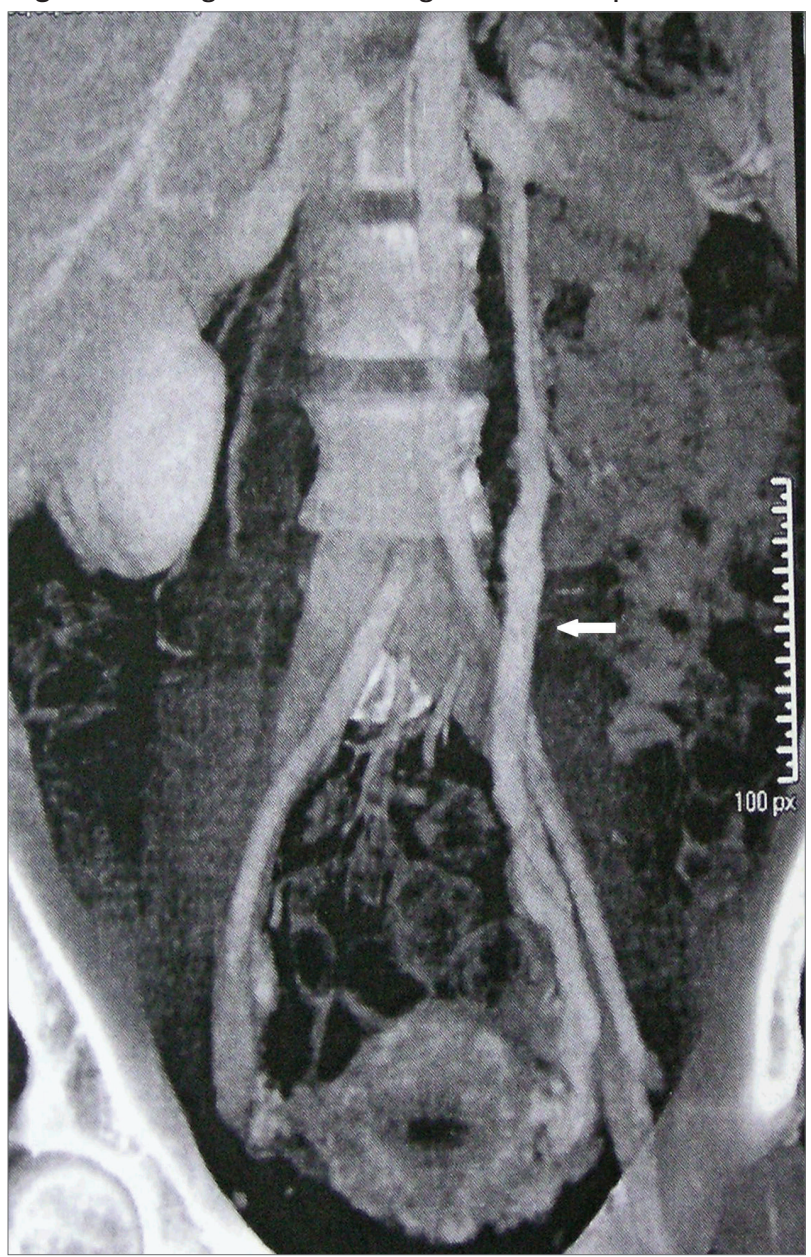


(novembro de 2009) (Figuras 1 a 3) mostrou compressão da veia renal esquerda no seu trajeto entre a artéria mesentérica superior e aorta abdominal, além de sugerir refluxo na veia ovariana esquerda, devido a importante dilatação apresentada por esta.

Com base na apresentação clínica da paciente e nos exames de imagem, a SQN foi diagnosticada.

\section{DIscUSSÃo}

A síndrome de compressão da veia renal esquerda pelas artérias aorta e mesentérica superior foi inicialmente descrita por El Sadr, ${ }^{3}$ sendo, porém, De Schepper quem a nomeou posteriormente "síndrome de quebra-nozes" (Nutcracker). ${ }^{4}$ Com provável e relativa prevalência superior no sexo feminino, ${ }^{1,5,6}$ postula-se que seja associada a situações particulares, como nefroptose ou escassez de gordura retroperitoneal, o que leva a um alongamento da veia renal e a uma diminuição do ângulo aortomesentérico, respectivamente. $\mathrm{Na}$ síndrome, há hipertensão da veia renal esquerda, ${ }^{7}$ e uma série de sinais e sintomas possíveis estão relacionados a essa condição, como hematúria, dor no flanco esquerdo, varicocele esquerda, proteinúria ortostática, fadiga crônica e congestão pélvica. O mecanismo mais provável da hematúria seria a consequente ruptura do fino septo que separa as veias do sistema coletor urinário. ${ }^{8}$

O diagnóstico da SQN ainda é de difícil realização, já que esses sintomas são relativamente comuns em outras situações clínicas, particularmente a nefrolitíase. Hematúria no orifício ureteral esquerdo, na ausência de qualquer outra patologia detectável, deve levantar a suspeita. ${ }^{9}$ No caso de nossa paciente, a cistoscopia não identificou essa particularidade. Ela pode ser de grande ajuda, porém é possível que não revele hematúria intermitente no momento do exame. ${ }^{2}$ Muitas vezes, o diagnóstico se dá após longa investigação, e, geralmente, determinados exames de imagem são essenciais, como a angioressonância, angiotomografia, ultrassonografia por Doppler, ou mesmo a venografia. ${ }^{7}$ Esses exames de imagem podem mostrar a compressão característica da síndrome, porém o diagnóstico é realizado apenas na presença de hematúria e hipertensão da veia renal esquerda associadas. ${ }^{7} \mathrm{Na}$ ausência dessa associação, trata-se do fenômeno de quebra-nozes. Essa classificação do paciente como sindrômico ou apresentando apenas o fenômeno é bastante discutível, pois não existe consenso sobre em qual grau os achados clínicos podem ser considerados relevantes. ${ }^{1}$ Estes podem variar de hematúria microscópica assintomática até severa congestão pélvica. ${ }^{10,11}$
Com base na hematúria sem causa estabelecida e nos resultados sem alterações nos exames de ultrassonografia abdominal e cistoscopia, a possibilidade de anormalidade vascular foi levantada. Uma angiotomografia foi solicitada, a qual, associada às manifestações clínicas, foi suficiente para o estabelecimento do diagnóstico neste caso. O fenômeno de quebra-nozes é diagnosticado quando uma redução do calibre da veia renal esquerda ultrapassa $50 \%$, enquanto esta cruza a aorta abdominal. ${ }^{12}$ A venografia é o método de imagem mais informativo, porém não é perfeitamente confiável. ${ }^{13}$ Em virtude disso - e em razão de sua maior invasividade -, tende a ser resguardada a casos específicos, não sendo comumente empregada, ou ainda, utilizada como último recurso., ${ }^{2,14}$

A ultrassonografia vascular por Doppler também é útil no estabelecimento do diagnóstico. ${ }^{2,10}$

O tratamento da SQN ainda é controverso, ${ }^{15}$ podendo ser conservador ou cirúrgico, relacionando-se intimamente à gravidade dos sintomas. $\mathrm{O}$ tratamento conservador é sugerido aos pacientes que apresentam sintomas toleráveis, como leve hematúria; ${ }^{16}$ já o tratamento cirúrgico é indicado nos casos de hematúria severa associada a anemia, insuficiência renal funcional, severa dor pélvica ou ineficácia de tratamento conservador após 24 meses de acompanhamento clínico. ${ }^{17-20}$

Alguns medicamentos têm sido investigados quanto a sua eficácia no controle dos sintomas da síndrome, ${ }^{21-23}$ apresentando resultados que devem despertar interesse para mais estudos a respeito de terapêuticas medicamentosas. O primeiro tratamento cirúrgico para a condição foi proposto por Pastershank, na década de $1970,{ }^{24}$ e, desde então, surgiram numerosas técnicas cirúrgicas para o tratamento.

A transposição da veia renal esquerda tem sido relatada como um procedimento cirúrgico seguro e efetivo. ${ }^{8,9}$

A simples nefropexia com excisão das varicosidades não é mais recomendada, pois não trata a situação fisiopatológica primária. $0^{9,25}$

O autotransplante renal é a técnica mais invasiva, porém está associada a baixas taxas de morbidade e excelentes resultados. Alguns autores acreditam que é o tratamento mais eficaz no que diz respeito à resolução dos sintomas da síndrome. ${ }^{25}$

O tratamento endovascular, por meio do uso de stent, é recente. Tem se mostrado eficaz e seguro, sendo uma opção a terapêuticas mais invasivas. ${ }^{26,27}$

Devido à redução na intensidade dos sintomas, a paciente encontra-se no momento em tratamento conservador. 


\section{REFERÊNCIAS}

1. Kurklinsky AK, Rooke TW. Nutcracker phenomenon and nutcracker syndrome. Mayo Clin Proc 2010;85:552-9.

2. Zhang H, Zhang N, Li M, et al. Treatment of six cases of left renal nutcracker phenomenon: surgery and endografting. Chin Med J (Engl) 2003;116:1782-4.

3. El-Sadr AR, Mina E. Anatomical and surgical aspects in the operative management of varicocele. Urol Cutaneous Rev 1950;54:257-62.

4. de Schepper A. "Nutcracker" phenomenon of the renal vein and venous pathology of the left kidney. J Belge Radiol 1972;55:507-11.

5. Ahmed K, Sampath R, Khan MS. Current trends in the diagnosis and management of renal nutcracker syndrome: a review. Eur J Vasc Endovasc Surg 2006;31:410-6.

6. Chen YM, Wang IK, Ng KK, Huang CC. Nutcracker syndrome: an overlooked cause of hematuria. Chang Gung Med J 2002;25:700-5.

7. Kim KW, Cho JY, Kim SH, et al. Diagnostic value of computed tomographic findings of nutcracker syndrome: Correlation with renal venography and renocaval pressure grandients. Eur J Radiol 2010. [Epub ahead of print]

8. Reed NR, Kalra M, Bower TC, Vrtiska TJ, Ricotta JJ 2nd, Gloviczki P. Left renal vein transposition for nutcracker syndrome. J Vasc Surg 2009;49:386-93.

9. Wang L, Yi L, Yang L, et al. Dianogsis and surgical treatment of nutcracker syndrome: a single-center experience. Urology 2009;73:871-6.

10. Shin JI, Park JM, Lee JS, Kim MJ. Effect of renal Doppler ultrasound on the detection of nutcracker syndrome in children with hematuria. Eur J Pediatr 2007;166:399-404.

11. Rogers A, Beech A, Braithwaite B. Transperitoneal laparoscopic left gonadal vein ligation can be the right treatment option for pelvic congestion symptoms secondary to nutcracker syndrome. Vascular 2007; 15:238-40.

12. Scholbach T. From the nutcracker-phenomenon of the left renal vein to the midline congestion syndrome as a cause of migraine, headache, back and abdominal pain and functional disorders of pelvic organs. Med Hypotheses 2007;68:1318-27.
13. Wolfish NM, McLaine PN, Martin D. Renal vein entrapment syndrome: frequency and diagnosis. A lesson in conservatism. Clin Nephrol 1986;26:96-100.

14. Thompson PN, Darling RC 3rd, Chang BB, Shah DM, Leather RP. A case of nutcracker syndrome: treatment by mesoaortic transposition. J Vasc Surg 1992;16:663-5.

15. Kim SH, Cho SW, Kim HD, Chung JW, Park JH, Han MC. Nutcracker syndrome: diagnosis with Doppler US. Radiology 1996;198:93-7.

16. Dever DP, Ginsburg ME, Millet DJ, Feinstein MJ, Cockett ATK. Nutcracker phenomenon. Urology 1986;27:540-2.

17. Stewart BH, Reiman G. Left renal venous hypertension "nutcracker" syndrome. Managed by direct renocaval reimplantation. Urology 1982;20:365-9.

18. Hohenfellner M, Steinbach F, Schultz-Lampel D, et al. The nutcracker syndrome: new aspects of pathophysiology, diagnosis and treatment. J Urol 1991; 146:685-8

19. Hohenfellner M, D’Elia G, Hampel C, Dahms S, Thüroff JW. Transposition of the left renal vein for treatment of the nutcracker phenomenon: long-term follow-up. Urology 2002;59:354-7.

20. Shokeir AA, el-Diasty TA, Ghoneim MA. The nutcracker syndrome: new methods of diagnosis and treatment. Br J Urol 1994;74:139-43.

21. Shin JI, Lee JS. ACE inhibition in nutcracker syndrome with orthostatic proteinuria: how about a hemodynamic effect? Pediatr Nephrol 2007;22:758.

22 Shin JI, Lee JS. Can chronic fatigue symptoms associated with nutcracker phenomenon be treated with aspirin? Med Hypotheses 2007;69:704-5.

23. Ha TS, Lee EJ. ACE inhibition can improve orthostatic proteinuria associated with nutcracker syndrome. Pediatr Nephrol 2006;21:1765-8.

24. Pastershank SP. Left renal vein obstruction by a superior mesenteric artery. J Can Assoc Radiol 1974;25:52-4.

25. Salehipour M, Rasekhi A, Shirazi M, Haghpanah A, Jahanbini S, Eslahi SA. The role of renal autotransplantation in treatment of nutcracker syndrome. Saudi J Kidney Dis Transpl 2010;21:237-41.

26. Kim JY, Joh JH, Choi HY, Do YS, Shin SW, Kim DI. Transposition of the left renal vein in nutcracker syndrome. Eur J Vasc Endovasc Surg 2006;31:80-2.

27. Hartung O, Grisoli D, Boufi M, et al. Endovascular stenting in the treatment of pelvic congestion caused by nutcracker syndrome: lessons learned from the first five cases. J Vasc Surg 2005;42:275-80. 